\title{
Child-Robot Spatial Arrangement in a Learning by Teaching Activity
}

\author{
Wafa Johal $^{1,2}$, Alexis Jacq ${ }^{1,3}$, Ana Paiva ${ }^{3}$ and Pierre Dillenbourg ${ }^{1}$
}

\begin{abstract}
In this paper, we present an experiment in the context of a child-robot interaction where we study the influence of the child-robot spatial arrangement on the child's focus of attention and the perception of the robot's performance. In the "Co-Writer learning by teaching" activity, the child teaches a Nao robot how to handwrite. Usually only face-to-face spatial arrangements are tested in educational child robot interactions, but we explored two spatial conditions from Kendon's Fformation, the side-by-side and the face-to-face formations in a within subject experiment. We estimated the gaze behavior of the child and their consistency in grading the robot with regard to the robot's progress in writing. Even-though the demonstrations provided by children were not different between the two conditions (i.e. the robot's learning didn't differ), the results showed that in the side-by-side condition children tended to be more indulgent with the robot's mistakes and to give it better feedback. These results highlight the influence of experimental choices in child-robot interaction.
\end{abstract}

\section{INTRODUCTION}

Application of robots in learning tasks has been increasing and has shown very promising results these past ten years. $R$-learning, in reference to e-learning, aims to design robots that would be helpful for learning. The potential of robots for individual adaptation and their physical ability makes them fit well in the educational world. We investigate how robots can bring added value for learning by designing and selecting relevant physical tas and setting up the appropriate interaction. In that vein, the Co-Writer projects aims to help children with difficulties in handwriting [3]. It is based on the idea of "learning by teaching". Under this paradigm, by teaching a robot (Nao from Aldebaran) how to write, children learn and improve their handwriting at the same time. The activity also plays with the protg effect, which makes children more engaged in practising for the robot than for themselves.

Previous studies in the Co-Writer project helped to develop a system that generates handwriting for the robot based on demonstrations from the child[3]. Case studies presented in [5] showed that children were able to stay engaged in long term interactions with repeated sessions within the Co-Writer activity in real pedagogical/therapeutic contexts. These works proved to have a positive effect on the extrinsic motivation of the children when practicing their handwriting, thanks to the protg effect.

Authors in [14] had a similar approach to learning by teaching with their Care Receiving Robot, who was being taken care of and taught by a child using physical interaction.

\footnotetext{
${ }^{1}$ CHILI Lab, ${ }^{2}$ LSRO Lab, École Polytechnique Fédérale de Lausanne (EPFL), Switzerland firstname. lastnamedepfl.ch

${ }^{3}$ Instituto Superior Técnico (IST), University of Lisbon, Portugal firstname. lastnamedinesc-id.pt
}

In this study, authors chose to investigate handwriting (or drawing shapes) as well, but with a more physical based approach. In their experimental setting, the child would teach handwriting to the robot by placing himself behind the robot and moving it's hand. This study varied from other works in educational human-robot interaction in a way that the child was not only facing the robot but would act as a care-taker rather than a teacher.

As robots are entering our living space, they must adapt to our social norms. These norms vary from politeness to unspoken social rules (as for instance, the personal space of a person). In home environments, robots will be expected to perform their functions in a manner that is clearly understandable and predictable by the humans around. This requires adaptive personalization of the robot to the individual needs of the humans, but also to the task being currently performed. Some previous studies showed that the spatial setting with a robot was also a way to convey non-verbal messages and it serves to influence the relationship with the user[17], [10]. Spatial arrangement is still a factor relatively unexplored in HRI and its influence on the interaction is still unclear.

In this study, we explore the effect of spatial arrangement on the child-robot interaction within the Co-Writer activity.

\section{RELATED WORKS}

Spatial configuration is part of non-verbal cues of interpersonal stance. Spatial arrangement is also a social signal that tells about the relationship between people. Kendon [7] called the F-Formation a spatial-orientational system that aims to explain how people arrange themselves in a group interaction. Kendon proposed three main F-formations : face-to-face (or vis-a-vis), L shape and side-by-side. These formations are illustrated by Figure 1.

According to [9], spatial arrangement and seating position can determine a person's role in a group. It could also predispose the person to either competitive (face to face) or cooperative (side by side) mental setting in a shared task.

In educational child-robot interaction, quite often, the robot is placed in front of the learner playing the role of a tutor or a peer helping the child, and uses social capabilities to enhance the learning [15]. Kanda [6] reported an experiment involving children that learns from a robot as they would from a peer. In this other experiment, [13] showed in a face-to-face setting that the physical presence of the robot produced measurable learning gains. [8] confirmed these results but recommended caution when applying social behaviors in a tutoring context. 


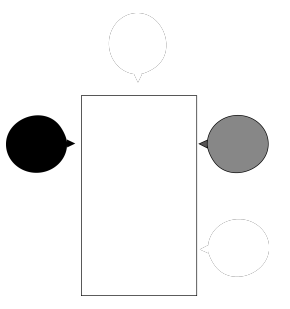

(a) Face to face

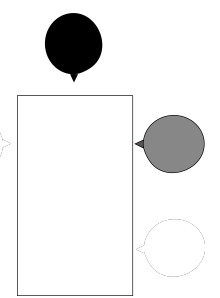

(b) L-Shape

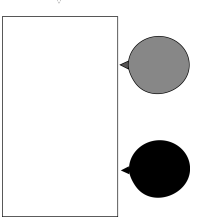

Fig. 1: Three possible (c) Kide by Side $^{\text {Siden }}$ F-formations around a rectangular table

Spatial arrangements have been studied in previous research[11], [4], [18] in HRI, often as a challenge of path planning, or as a metric but less frequently as a non-verbal cue of communication in a learning task.

We propose to study the impact of spatial arrangement on the engagement of children in a handwriting task. We performed a study in a school with 12 children performing the same teaching task under two conditions. The child was either facing the robot (similar to other tutoring experiments) or was sitting next to the robot (as a peer teaching setup).

\section{METHOD}

The experiment took place in a primary school in Switzerland where children are taught in English. In this experiment, we targeted children aged 5 y.o. who start handwriting, but do not typically master it yet. The children interacted with the robot under two conditions of the F-Formation in a counterbalanced manner.

Our goal was to investigate the effect of spatial arrangement on the interaction. We expected that children would give better feedback(see III-C) to the robot when teaching it in a side-by-side configuration for several reasons. The sideby-side arrangement corresponds to a cooperative arrangement which is close to a peer teaching setting, unlike the face-to-face teaching arrangement, which is more frequent seen in competitive or conversation tasks (closer to a teacherstudent relationship). Also, in the side-by-side formation, the robot and the child have the same visual perspective of the shared tablet on which they write. Perspective sharing is an ability that facilitates mutual understanding [1]. Hence by having the robot and the child side-by-side, higher mutual understanding would be expected.

\section{A. Participants and Apparatus}

12 subjects (six girls) from the same classroom (aged 5 to 6 y.o.) participated to the within subject study. The two considered F-formations for this experiment are presented in the Figure 2: face-to-face $2 \mathrm{a}$ and side-by-side $2 \mathrm{~b}$. Children were presented the two conditions sequentially with and interval of three days in a counterbalanced manner.
Apart from this change in the spatial setting, the interaction was kept the same. The children were told they had to teach the robot how to write some words. We briefly presented the two tablet interfaces and the interaction started. Any word from a list displayed on the selection tablet could be picked by the child. As the robot would start to write this word. It was set to be a very bad writer at the beginning of the first session for each child. The child could then give a feedback to the robot by pressing thumbs up or a thumbs down buttons how many times they wanted. The child would then use a pen and demonstrate how to write the word and the robot would then rewrite the word using the demonstration of the child. The generated writing of the robot was computed to be halfway from its previous writing state and the new demonstration provided by the child.

Several hypotheses were made concerning the influence of the spatial arrangement on the interaction. We expected that the gaze behavior of the child would vary according to the spatial configuration. More gazing at the robot's head would be expected in the face-to-face condition. Our research question was to measure the degree to which this also influenced the way children behaved as a teacher (were they more or less severe with the robot facing them). As children give a feedback to the robot for each demonstration, we intend to measure if there is any difference between vis-avis and side-by-side regarding this feedback (does the sideby-side condition trigger more positive feedback? or more appropriate feedback?).

The degree of engagement of the child in the task can also be influenced by the arrangement. For that particular aspect, we will measure the number of repetitions of each word, as well as the with-me-ness, which is discussed in the following section. Since children were quite young, we choose to not use any self-reported measures or questionnaires.

\section{B. With-me-ness}

The with-me-ness introduced in HRI by [12], helps to set specific targets during each state of the interaction and to determine whether the user is looking at one of these attentional targets or not. The algorithm is based on the $d$-lib library that helps to estimate the head pose of the user using a video from a webcam device for instance. From this head pose, the visual focus of attention (VFoA) is computed. The inclusion of the targets in the VFoA allows then to compute the with-me-ness. In a simple way, the with-me-ness value will increase if the child looks at the specific set of targets, or else it decreases. This measure allows us to see if the child is engaged in the interaction and is looking at the tablet or the robot's head when he/she is expected to (according to the task). Indeed, in our learning by teaching activity, the robot has also a hidden pedagogical role. It's progress aim actually to make the child practice and think about his/her own way of writing. In that sense, we can set attentional targets as proposed by [16] when the with-me-ness was first introduced to measure learner's attention to teachers in MOOC videos.

This measure is actually very close to the notion of synchrony already studied in HRI [2], where bounding between 


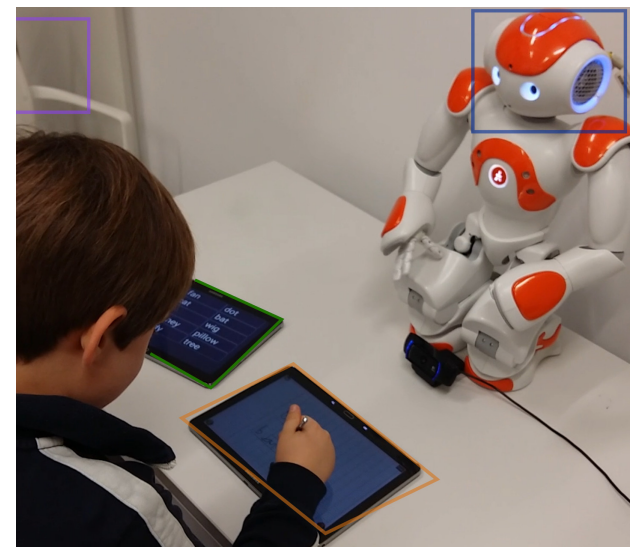

$\begin{array}{ll}\text { (a) Face to face spatial formation } & \text { (b) Side by Side spatial formation } \\ \text { Fig. 2: Experimental set-ups showing the with-me-ness targets in the rectangles: orange - the writing tablet, blue - the robot's }\end{array}$

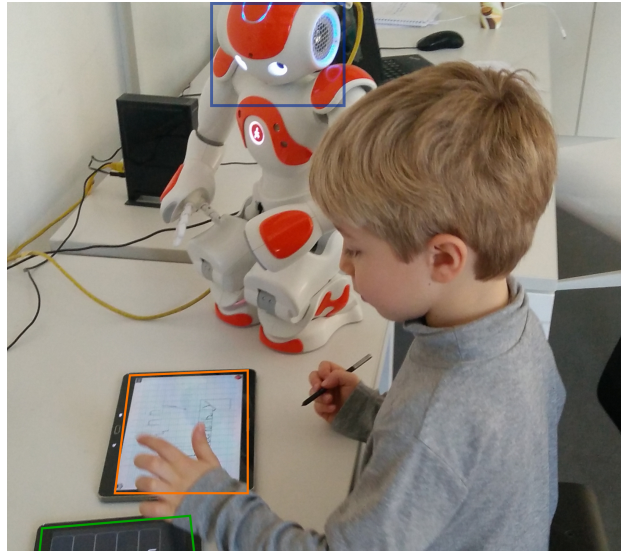

Fig. 2: Experimental set-ups showing the with-me-ness targets in the rectangles: (b) Side by Side spatial formation head, green - the tablet used to select a word to teach to the robot

individuals is reflected by their ability to synchronize in the task (look at the same time at the same targets).

For this experiment, the targets were: "the observer"(a teacher or a teacher assistant),"the experimenter", "the selection tablet"(the tablet used to pick a word) and the "tablet". The with-me-ness is initially set to 0.5 and takes values from 0 to 1 . According to the state in which the activity is, (robot is writing, child is writing,...) the with-me-ness will be increased if the child looks at a target that is in the set of task-related targets (expected within this particular state of the activity). We record these targets and the with-me-ness at a frequency of about $1 \mathrm{~Hz}$. The evolution of the with-meness is computationally attenuated in order to remove noisy data (by using the weighted cumulative of the with-me-ness value).

The targets were defined according to their spatial relation with the camera used (placed on the table at the robot's feet). The position of the targets was changed according to the F-formation condition, but the camera stayed at the same position Figure 2 shows these targets for the two conditions.

\section{Reward Mechanism}

The tablet interface on which the child and the robot practice their handwriting shows two buttons that allow the child to give a positive (green thumb-up) or negative (red thumb-down) feedback to the robot's handwriting. After every trial of the robot, the child could click on these feedback buttons as much as he/she wants. We monitored each of these clicks.

These clicks aimed to assess the child's perception of the robot's progress. When converging to a better writing we expect the child to give better feedback. However, these buttons could also be used by the child as an encouragement method and children could give a positive feedback even though the robot didn't make progress.

\section{Performances}

As the child was managing which word the robot would learn, he/she could also provide as many demonstrations as he/she wanted. The child was also free to change the word when satisfied with the robot's performance.
1) Response Time and Writing Time: We recorded the time spent on the writing and the response time for each word demonstrated by the children. We also monitored the number of demonstrations provided by the child for each word. These measure were cues to how dedicated the child was in the task

2) Writing Score for the Robot: Since the learning algorithm took as input the child's demonstration, if the child provided repeatedly the exact same demonstration, then the robot would converge faster to his/her handwriting. This score is hence a hint on children's regularity, with the underlying assumption that the regularity in handwriting is a sign of legibility.

At each demonstration of the robot, we were calculating a writing score. Each demonstration was encoded as a list of seventy 2D points. This writing score is the euclidian distance between the demonstrated letter by the child and the generated letter by the robot.

We also computed the evolution of this score at each demonstration. We represented the evolution of the robot's handwriting with different states: 'S=': the first trial of this word by the robot, 'S-': the score is decreasing and 'S+' the score is increasing. If the child was changing a lot his/her way of writing between consecutives demonstration, the score would then decreasee. In the contrary, the regularity of the demonstration would make the score increase rapidly.

\section{RESULTS}

\section{A. Reward Mechanism}

Children gave feedback with an average of 3 feedbacks per demonstration (i.e. per interaction loop). We summed the feedbacks for each demo with positive feedback counted as +1 and negative as -1 . Figure 3 shows the average evolution along the demonstrations of the sum of feedbacks given for all children and for both spatial condition. We noticed that the feedback is first negative and grows towards a positive feedback after each demonstration. In average for all the children, the sum of feedbacks stayed negative until the 5 th demonstration. Children well understood that they were teaching the robot and often gave bad scores for the first 


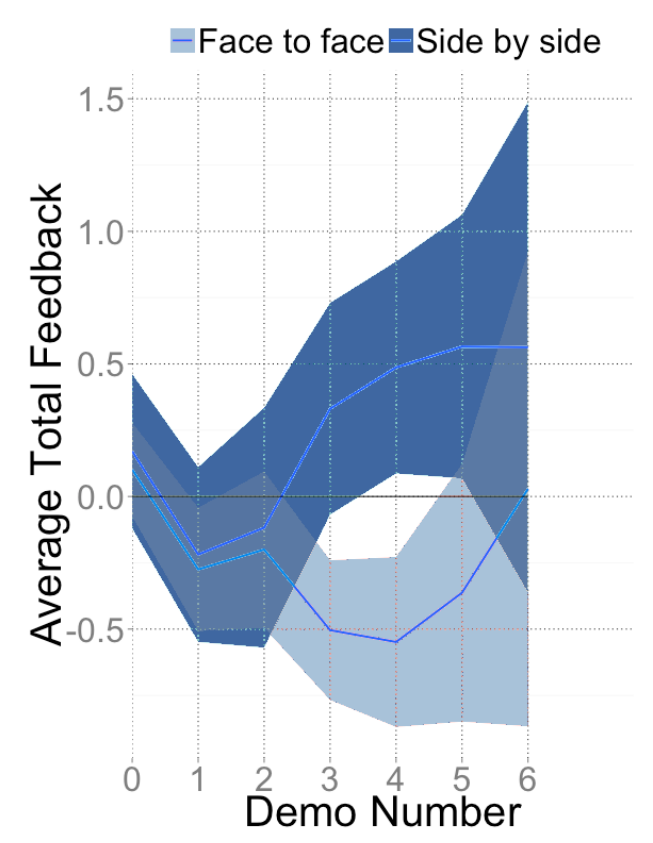

Fig. 3: Evolution of Feedback along the demonstration index(Mean: line, Confidence Interval 0.95: filled area)

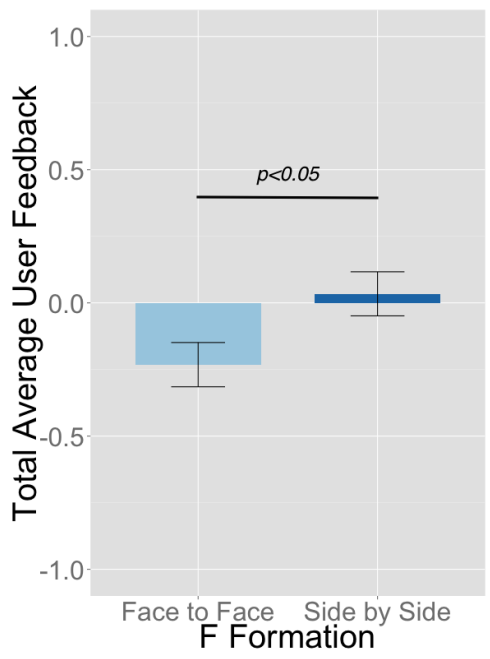

Fig. 4: Feedback Sum According to the F-Formation (Means and Confidence Intervals)

untrained trial of the robot. Children usually gave a positive feedback just before changing the word taught to the robot.

Even though the average feedback was increasing along with the number of demonstration for both condition, they don't seems to increase the same way(see Figure3) The effect of the F-Formation on the feedback from the child was statistically significant (Anova Repeated measures within subjects: $\left.F_{1,272}=4.396, p<0.05\right)$. As, illustrated on Figure 4 , the average feedback per demonstration was greater $M=$ $0.03, S D=1, N=147)$ for the side-by-side condition compare to the face-to-face condition $(M=-0.23, S D=$ $0.98, N=138$ ).

Children kept giving feedback along the interaction and no drop in the number of feedbacks was observed during the

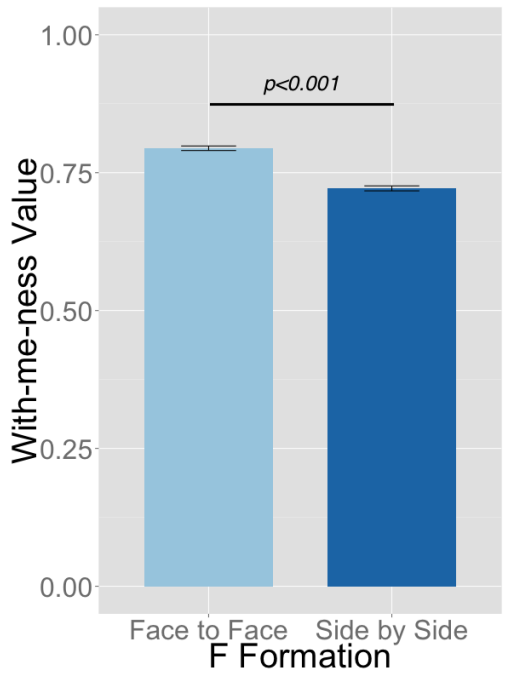

Fig. 5: With-me-ness According to the F-Formation (Means and Confidence Intervals)

experiment. They took their duty to teach the robot seriously in that way.

We also observe an order effect showing that children tended to give higher feedback in the second session compare to the first one. This can simply be explained by the fact that the robot's learning state was progressive between the two session. The robot didn't start to learn from scratch in the second session but had already some knowledge from the first session with this same child.

\section{B. With-me-ness}

Children understood the dynamics of the interaction, as in general the with-me-ness stayed above 0.5 throughout the interaction (started et 0.5 but always finished above). The effect of the F-Formation on the with-me-ness of the child was statistically significant (Anova Repeated measures within subjects: $\left.F_{1,15983}=293.2, p<0.001\right)$. The with-meness was greater $(M=0.79, S D=0.18, N=7722)$ for the face-to-face condition compare to the side-by-side condition ( $M=-0.72, S D=0.21, N=8267$ ) (see Figure 5). This result was expected, as the robot was facing the robot, its face was more visible for the child.

Again, we observed an order effect with the with-me-ness increasing between the two sessions. As children were more comfortable with the system, it is logic that they started to learn the dynamics of the interaction knowing when to look at the selection tablet, the writing tablet and the robot.

\section{Performances}

1) Response Time and Writing Time: Figure 6 shows on the left the average number of demonstration for each word taught to the robot for the two spatial arrangement conditions. There was no significant difference between the conditions even-though the average number of demonstration given by the child in the side-by-side condition seems higher than the face-to-face.

The center graph of Figure 6 shows the average response time for the demonstration provided by the children for the 

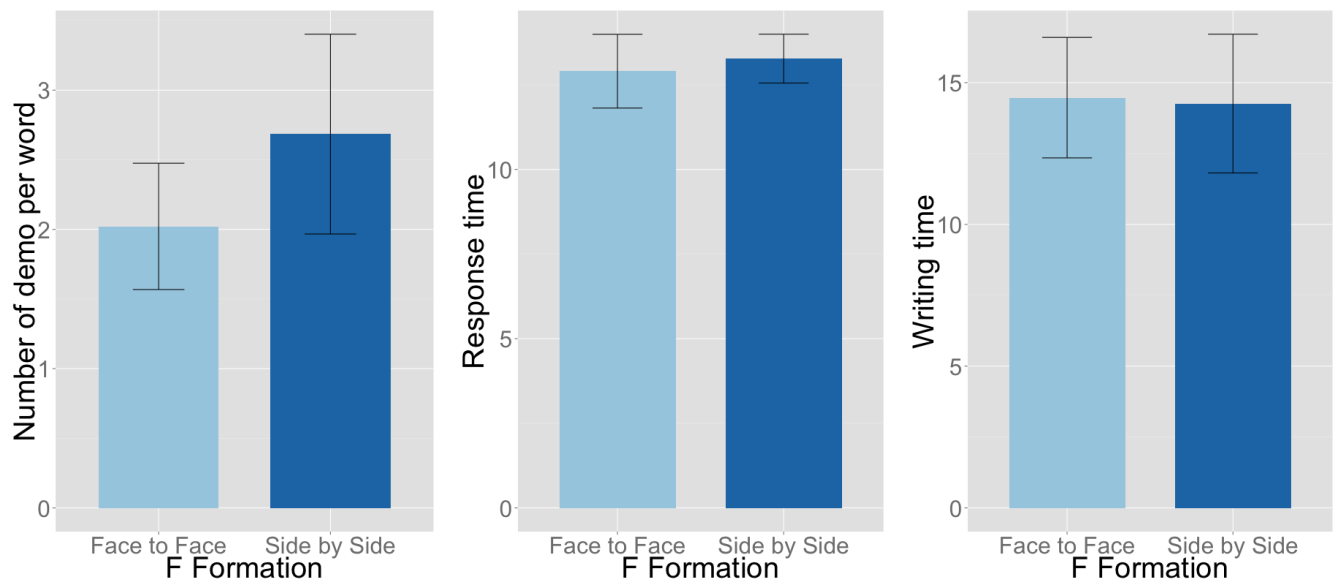

Fig. 6: Number of demonstration per word (left), response time (center), writing time (right) - (Means and Confidence Intervals)

two spatial arrangement conditions. The response time is the delay between the time when the robot finishes to write and the time when the child touches the tablet. There was no significant difference between the conditions.

The writing time (right graph on Figure 6) corresponds to the delay between the time when the robot finishes its trial and the time the child finishes its new demonstration or changes word. This time also include the moment in which the child can give a feedback via the buttons. No significant difference in the writing time was found.

These results show that children when not spending more time per word in one condition of the other. The spatial condition didn't influence the involvement of the child in the task.

2) Writing Score for the Robot: There was no significant difference of writing score in the F-formation condition tested (side-by-side: $M=0.80, S D=0.08, N=135$, faceto-face: $M=0.79, S D=0.08, N=191$, Anova Repeated measures within subjects: $p>0.1$ ). This result means that the children were teaching as well in the face-to-face condition as in the side-by-side condition. However, results showed significant differences in terms of feedback given to the robot regarding the score of the robot.

We analyzed the probabilities of succeeding events considering feedback events and score evolution events.

TABLE I: Probability of feedback given the score per word in the two conditions : face-to-face / side-by-side

\begin{tabular}{|c|c|c|c|}
\hline $\begin{array}{ll}\text { Score Event } & \text { Feedback }\end{array}$ & Positive & Negative & None \\
\hline Score Increases & $0.37 / 0.44$ & $0.39 / 0.27$ & $0.24 / 0.29$ \\
\hline Score Decreases & $0.28 / 0.47$ & $0.36 / 0.20$ & $0.36 / 0.33$ \\
\hline
\end{tabular}

Table I shows the probabilities of transition of feedback events (positive or negative) after the score increases or after the score decreases (computed using Markov Chain). We notice that in general the positive feedback probabilities are higher for the side-by-side condition in comparison to the face-to-face. On the contrary, the probability of having a negative feedback after is higher in the face-to-face con- dition. We can also notice that when the score decreases, the probability of having a positive feedback is almost twice higher in the side-by-side condition (total number of events $=$ ).

All the transitions between the scores and the feedback buttons are illustrated on Figure 7. Children were displaying a more positive attitude towards the robot when placed in side-by-side position even when the robot was not improving. This positive attitude was showed by rewarding more improvements of the robot and also penalizing less the retrogression of the robot's writing. Children even rewarded retrogression more often in the side-by-side condition. The reward given by children showed to be not often appropriate. For instance in the face-to-face condition, score increasing got more than a third of the time given a negative feedback. These differences in the transition matrix were not significant (Pearson's Chi-squared test, $X-$ squared $=30, d f=$ $25, p-$ value $=0.2243)$ and a study with a larger number of participants might have given more precise results.

\section{DISCUSSIONS AND CONCLUSIONS}

In this paper we presented a study that aimed to elicit the effect of spatial arrangement on a learning task between a robot and a child. Spatial configuration has never to our knowledge been studied in terms of effect on the task but usually in the context of the social interaction. In this experiment, we showed that this spatial arrangement could also have an influence in the task and on how the child was behaving as a teacher. Our results showed that even if the robot's learning didn't vary between the side-by-side and the face-to-face condition, the feedback from the child was varying. This suggests that the relationship between the child and the robot was different between the conditions. These results are in line with the literature in spatial arrangement and non-verbal social cues.

While running the experiments, we noticed that the children were expecting the robot to react to the feedback, and the robot was moving only when writing. We are working on different form of reactions for future experiments to allow the robot to react according to the feedback from the child and 


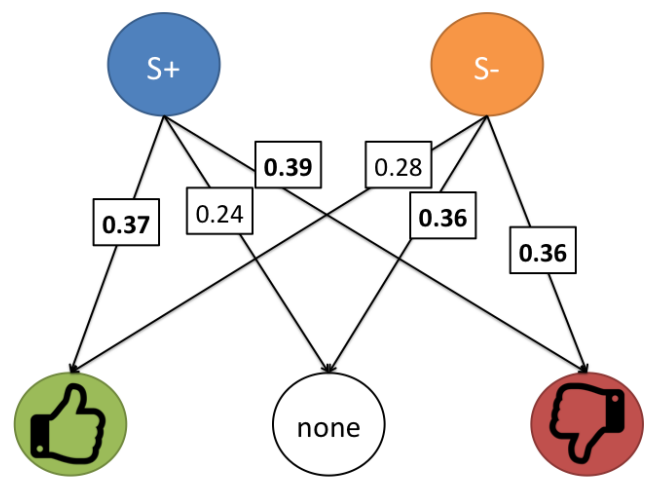

Fig. 7: Transitions states from score evolutions $(S+$ : score increasing compared to previous score, $S-:$ score decreasing

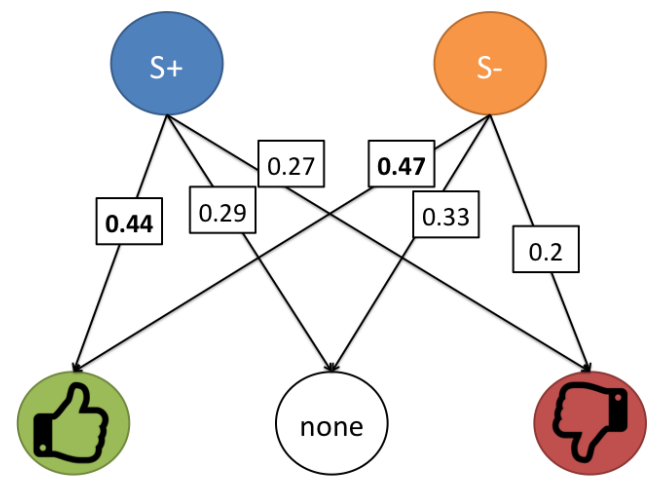

Fig. 7: Transitions states from score evolutions $(S+$ : score increasing compared to previous score, $S-:$ score decreasing compare to the previous) to children's feedback (thumb up: positive feedback, thumb down : negative feedback, and no feedback given)

also in perspective with the fairness of the feedback regarding the score of the robot. For instance, if the child gives a positive feedback when the robot is actually improving, the robot should display a positive emotion. If it is given a positive feedback but does not actually make any progress, it might express doubt to force the child to be more exigent.

Even if our results showed that the side-by-side elicited more positive feedback from the children, statistical significance was not reached, and if the robot would display some emotional reaction during the interaction, the same effect might not be observed. We plan to enhance the interaction with some behavioral reaction and to research the effect of such reactions on the child's perception of the robot's progresses. Long term studies might also bring more insights to theses results in order to see if the effect of the spatial arrangement sustain over time.

To conclude, we believe that spatial arrangement can be used as a non-verbal cue of communication to contextualize the interaction (as cooperative or competitive for instance). We also believe that choices in terms of spatial arrangements in HRI studies should be thoroughly made as they may influence the interaction.

\section{ACKNOWLEDGMENT}

This research was partially supported by the Fundação para a Ciência e a Tecnologia (FCT) with reference UID/CEC/ 50021/2013, and by the Swiss National Science Foundation through the National Centre of Competence in Research Robotics.

\section{REFERENCES}

[1] M. Berlin, J. Gray, A. L. Thomaz, and C. Breazeal. Perspective taking: An organizing principle for learning in human-robot interaction. 2006.

[2] E. Delaherche, M. Chetouani, A. Mahdhaoui, C. Saint-Georges, S. Viaux, and D. Cohen. Interpersonal synchrony: A survey of evaluation methods across disciplines. Affective Computing, IEEE Transactions on, 3(3):349-365, 2012.

[3] D. Hood, S. Lemaignan, and P. Dillenbourg. When children teach a robot to write: An autonomous teachable humanoid which uses simulated handwriting. In Proceedings of the 2015 ACM/IEEE Human-Robot Interaction Conference, 2015.

[4] H. Hüttenrauch, K. S. Eklundh, A. Green, E. Topp, et al. Investigating spatial relationships in human-robot interaction. In Intelligent Robots and Systems, 2006 IEEE/RSJ International Conference on, pages 5052-5059. IEEE, 2006.
[5] A. Jacq, S. Lemaignan, F. Garcia, P. Dillenbourg, and A. Paiva Building successful long child-robot interactions in a learning context. In Proceedings of the 2016 ACM/IEEE Human-Robot Interaction Conference, 2016.

[6] T. Kanda, T. Hirano, D. Eaton, and H. Ishiguro. Interactive Robots as Social Partners and Peer Tutors for Children: A Field Trial. HumanComputer Interaction, 19(1):61-84, 2004.

[7] A. Kendon. Spacing and orientation in co-present interaction, pages 1-15. Springer, 2010.

[8] J. Kennedy, P. Baxter, and T. Belpaeme. The Robot Who Tried Too Hard: Social Behaviour of a Robot Tutor Can Negatively Affect Child Learning. Proceedings of the ACM/IEEE International Conference on Human-Robot Interaction, pages 67-74, 2015.

[9] M. Knapp, J. Hall, and T. Horgan. Nonverbal communication in human interaction. Cengage Learning, 2013.

[10] A. Kristoffersson, K. S. Eklundh, and A. Loutfi. Measuring the quality of interaction in mobile robotic telepresence: a pilots perspective International Journal of Social Robotics, 5(1):89-101, 2013.

[11] H. Kuzuoka, Y. Suzuki, J. Yamashita, and K. Yamazaki. Reconfiguring spatial formation arrangement by robot body orientation. In Proceedings of the 5th ACM/IEEE International Conference on Human-robot Interaction, HRI '10, pages 285-292, Piscataway, NJ, USA, 2010. IEEE Press.

[12] S. Lemaignan, F. Garcia, A. Jacq, and P. Dillenbourg. From realtime attention assessment to with-me-ness in human-robot interaction. In Proceedings of the 2016 ACM/IEEE Human-Robot Interaction Conference, 2016.

[13] D. Leyzberg, S. Spaulding, and B. Scassellati. Personalizing robot tutors to individuals' learning differences. Proceedings of the 2014 ACM/IEEE international conference on Human-robot interaction HRI '14, pages 423-430, 2014.

[14] S. Matsuzoe and F. Tanaka. How smartly should robots behave?: Comparative investigation on the learning ability of a care-receiving robot. Proceedings - IEEE International Workshop on Robot and Human Interactive Communication, pages 339-344, 2012.

[15] M. Saerbeck, T. Schut, C. Bartneck, and M. D. Janse. Expressive robots in education: varying the degree of social supportive behavior of a robotic tutor. Proceedings of the 28th international conference on Human factors in computing systems - CHI '10, pages 1613-1622, 2010.

[16] K. Sharma, P. Jermann, and P. Dillenbourg. with-me-ness: A gazemeasure for students attention in moocs. In International conference of the learning sciences, number EPFL-CONF-201918, 2014.

[17] L. Takayama and C. Pantofaru. Influences on proxemic behaviors in human-robot interaction. 2009 IEEE/RSJ International Conference on Intelligent Robots and Systems, IROS 2009, (2009):5495-5502, 2009.

[18] M. Vázquez, A. Steinfeld, S. E. Hudson, and J. Forlizzi. Spatial and other social engagement cues in a child-robot interaction: Effects of a sidekick. In Proceedings of the 2014 ACM/IEEE international conference on Human-robot interaction, pages 391-398. ACM, 2014. 